\title{
THE ADDITIVE GROUP OF COMMUTATIVE RINGS GENERATED BY IDEMPOTENTS ${ }^{1}$
}

\author{
PAUL HILL
}

\begin{abstract}
If $R$ is a ring, let $R^{+}$denote its additive group. Our purpose is to give an elementary proof that if $R$ is a commutative ring generated by idempotents, then any subring of $R$ generated by idempotents is pure. This yields immediately an independent proof of the following result of G. M. Bergman. If $R$ is a commutative ring with identity and if $R$ is generated by idempotents, then $R^{+}$is a direct sum of cyclic groups.
\end{abstract}

G. M. Bergman [1, Corollary 4.3] has proved (in particular) that if $R$ is a commutative ring with identity generated by idempotents, then $R^{+}$is a direct sum of cyclic groups. This result is rather striking inasmuch as it contains, as a special case when $R^{+}$is torsion free, the recent celebrated result of G. Nöbeling [2] that the finite-valued functions from any set $I$ to the integers $Z$ form a free abelian group with respect to pointwise addition. Recall that the latter result was first proved for a countable set $I$ by E. Specker [3] with the aid of the continuum hypothesis.

If $S$ is a subset of the ring $\mathrm{R}$, we shall use $\{S\}$ to denote the subring generated by $S$, while $\langle S\rangle$ denotes the subgroup of $R^{+}$that $S$ generates. An elementary but important fact concerning a commutative ring $R$ generated by idempotents is the following. If $A$ is a finitely generated subring of $R$, then $A^{+}$is a finitely generated subgroup of $R^{+}$. Since a subgroup of a finitely generated commutative group is again finitely generated and since each element in $R$ is a linear combination over $Z$ of idempotent elements, it suffices to prove the above statement for the case that $A$ is generated as a ring by a finite number of idempotents. However, in this case, $A^{+}$is generated by all possible products of the idempotents generation $A$. Hence $A^{+}$is finitely generated.

THEOREM 1. Let $R$ be a commutative ring generated by idempotents. Any subring of $R$ generated by idempotents is pure.

Received by the editors June 6, 1972.

AMS (MOS) subject classifications (1970). Primary 20K10, 20K20, 20K25; Secondary 16A32.

Key words and phrases. Specker theorem, Nöbeling theorem, direct sum of cyclic groups, pure subgroups, commutative rings, idempotent generators.

1 This research was supported, in part, by NSF grant GP-29025.

(c) American Mathematical Society 1973 
THEOREM 2 (BERGMAN). If $R$ is a commutative ring generated by idempotents, then $R^{+}$is a direct sum of cyclic groups.

Proof of Theorem 1. Let $A$ be a subring generated by idempotents. The purity of $A$ in $R$ means that, for each prime $p$ and positive integer $n$, the equation $p^{n} x=a$ where $a \in A$ has a solution in $A$ whenever the equation has a solution in $R$. Thus it obviously suffices to prove the purity of $A$ in case the subring $A$ is generated (as a ring) by a finite number of idempotents $e_{1}, e_{2}, \cdots, e_{n}$. The purity of $A$ is accomplished by induction on $n$. Equivalently, for an idempotent $e$, we shall prove that $\{A, e\}$ is pure under the assumption that $A$ is a subring generated by $n$ idempotents and that $A$ is pure for any such subring. We can take $A=0$ to prove inductively the purity of a subring generated by a single nonzero idempotent.

For simplicity of notation, let $B=\{A, A e\}$ and let $C=\{B, e\}=\{A, e\}$. Since $A(A e) \subseteq A e$ and since $B e \subseteq B$, we observe that $B=\langle A, A e\rangle$ and $C=$ $\langle B, e\rangle$. In order to prove that $C$ is pure, we shall first prove that $B$ is pure. For a prime $\mathrm{p}$ and a positive integer $n$, suppose that $p^{n} x=b$ where $b \in B$ and $x \in R$. Since $B=\langle A, A e\rangle$, we can write $b=a_{1}+a_{2} e$ where $a_{1}, a_{2} \in A$. Since $p^{n}(x e)=b e=\left(a_{1}+a_{2}\right) e$ is contained in $A e$ and since $A e$ is a subring of $R$ generated by the $n$ idempotents $e_{1} e, e_{2} e, \cdots, e_{n} e$, the induction hypothesis asserts that $p^{n}\left(a_{3} e\right)=b e$ for some $a_{3} \in A$. Note that $p^{n}\left(x-a_{3} e\right)=$ $a_{1}-a_{1} e$. Let $D=\left\{e_{i}-e_{i} e\right\}$ be the subring of $R$ generated by the idempotents $e_{i}-e_{i} e$ where $1 \leqq i \leqq n$. Since $A=\left\{e_{1}, e_{2}, \cdots, e_{n}\right\}$ and since $a_{1} \in A$, the verification that $a_{1}-a_{1} e$ is contained in $D$ is trivial if we make the observation that $\Pi e_{i}-\left(\prod e_{i}\right) e=\prod\left(e_{i}-e_{i} e\right)$, where $\Pi$ represents a product over any nonempty subset of $[1,2, \cdots, n]$. By the induction hypothesis, we conclude that $p^{n} d=p^{n}\left(x-a_{3} e\right)$ for some $d \in D$ since $D$ is generated by $n$ idempotents and since $p^{n}\left(x-a_{3} e\right)=a_{1}-a_{1} e$ is in $D$. Thus $p^{n} x=p^{n}\left(a_{3} e+d\right)$, and we have proved the purity of $B$ in $R$ since $a_{3} e+d \in B$.

Based on the purity of $B$, we can now prove the purity of $C$. Suppose that $p^{n} x=c$ where $c \in C$ and $x \in R$. We want to show that $p^{n} c_{1}=c$ for some $c_{1} \in C$. Write $c=b+p^{m} q e$ where $b \in B$ and $(q, p)=1$. Since $(q, p)=1$, $q$ is not relevant to the equation under consideration and therefore there is no loss of generality in assuming that $q=1$, so now let $c=b+p^{m} e$. If $m \geqq n$, then $p^{n} b_{1}=b$ for some $b_{1} \in B$ due to the purity of $B$. Thus $c_{1}=b_{1}+$ $p^{m-n} e$ is the desired solution, $p^{n} c_{1}=c$. Therefore, we may assume that $m<n$. Hence let $r=n-m>0$. From the purity of $B$, we can choose $b_{1} \in B$ so that $p^{m} b_{1}=b$. Suppose, for a positive integer $i$, that we have already shown (as we have for $i=1$ ) the existence of $x_{i} \in\{x\}$ and $b_{i} \in B$ such that

$$
p^{r i+m} x_{i}=p^{m} b_{i}+p^{m} e .
$$


Since $e$ is idempotent, we obtain from (1) the equation

$$
p^{r i} x_{i}\left(p^{m} e\right)=p^{m} b_{i} e+p^{m} e
$$

and finally

$$
p^{r(i+1)+m} x_{i+1}=p^{m} b_{i+1}+p^{m} e,
$$

where $x_{i+1}=p^{r(i-1)} x_{i}^{2}$ and $b_{i+1}=b_{i}^{2}+2 b_{i} e$. Therefore, (1) has a solution, for all $i \geqq 1$, with $x_{i} \in\{x\}$ and $b_{i} \in B$. This implies that $p^{m} e+B^{+}$has infinite $p$-height in $\{C, x\}^{+} / B^{+}$. From a remark in the introduction, we know that $\{C, x\}^{+}$is a finitely generated group. Hence $\{C, x\}^{+} / B^{+}$is a direct sum of cyclic groups since it is finitely generated. We conclude that $p^{m} e+B^{+}$has finite order $t$ where $(t, p)=1$ since a direct sum of cyclic groups has no elements of infinite $p$-height except elements of finite order relatively prime to $p$. Since $(t, p)=1$, we may replace the equation $p^{n} x=$ $b+p^{m} e$ by the equation $p^{n} y=t b+t p^{m} e$. However, the latter equation has a solution in $B$ since $t p^{m} e \in B$ and $B$ is pure. We have shown that $C$ is pure, and this completes the proof of Theorem 1 .

The proof of Theorem 2 now follows quickly by induction on the (minimal) cardinality of a generating set of idempotents. Let $E$ be a set of idempotents that generate the ring $R$, and assume that $E$ has been chosen so that $|E|$ is as small as possible. If $E$ is finite, then $R^{+}$is finitely generated and, therefore, is a direct sum of cyclic groups. Thus assume that $|E|=\aleph \geqq \boldsymbol{\aleph}_{0}$. In view of the purity of any subring of $R$ generated by idempotents and the fact that direct sums of cyclic groups are the pureprojectives in the category of abelian groups, it suffices to prove that $\{A, e\}^{+} / A^{+}$is a direct sum of cyclic groups whenever $e$ is an idempotent and $A$ is a subring of $R$ generated by idempotents. The point here is that we can ascend to $R$ with a chain

$$
0=A_{0} \subset A_{1} \subset \cdots \subset A_{\alpha} \subset \cdots
$$

of subrings such that: (1) $A_{\alpha+1}$ is a simple extension of $A_{\alpha}$ by an idempotent, (2) $A_{\beta}=\bigcup_{\alpha<\beta} A_{\alpha}$ if $\beta$ is a limit, and (3) $A_{\alpha}$ is generated by fewer than $\boldsymbol{N}$ idempotents. To prove that $\{A, e\}^{+} / A^{+}$is a direct sum of cyclic groups, we again insert $B=\{A, A e\}$. Since $B=\langle A, A e\rangle$, we have the isomorphism $B^{+} \mid A^{+} \cong(A e / A \cap A e)^{+}$because $A \cap A e$ is an ideal of $A e$. Moreover, $A e / A \cap A e$ is a commutative ring generated by fewer than $W$ idempotents, for by hypothesis the same is true of $A$. By the induction hypothesis, $(A e / A \cap A e)^{+}$is a direct sum of cyclic groups. Since $B$ is pure in $C=$ $\{A, e\}=\langle B, e\rangle$ and since $C^{+} \mid B^{+} \cong\langle e\rangle^{+} /(B \cap\langle e\rangle)^{+}$is cyclic, $B^{+}$is a direct summand of $C^{+}$. Therefore, $C^{+} / A^{+}$is a direct sum of cyclic groups since $B^{+} / A^{+}$is. This completes the proof of Theorem 2. 


\section{REFERENCES}

1. G. Bergman, Boolean rings of projection maps, J. London Math. Soc. (2) 4 (1972), 593-598.

2. G. Nöbeling, Verallgemeinerung eines Satzes von Herrn E. Specker, Invent. Math. 6 (1968), 41-55. MR 38 \#233.

3. E. Specker, Additive Gruppen von Folgen ganzer Zahlen, Portugal. Math. 9 (1950), 131-140. MR 12, 587.

Department of Mathematics, Florida State University, Tallahassee, Florida 32306 